\title{
Editorial
}

\section{Public Health Practice and Management: Generation Next Oral Healthcare Leaders}

Public health practice and program management is intended to train skilled dental professionals to presuppose management roles as members of multidisciplinary healthcare teams. A degree in this specialty incorporates to develop, execute and assess effective public health projects. This program will also able to generate and analyze programs in the framework of the communities they serve, taking into account cultural, social, economic, ethical and legal factors. There is wide-ranged breadth of study in areas, such as health services research and policy, health and public policy, health economics

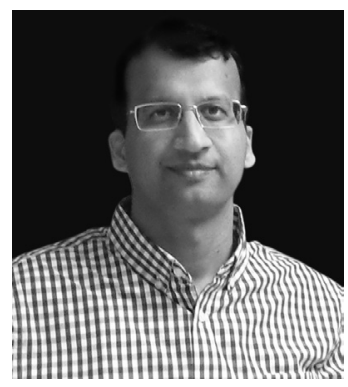
and policy, bioethics and health policy, health leadership and management and social policy.

An expert in dental public health practice and management will have a perceptive of health issues and be able to apply concepts involved in the planning, implementation, function, assessment of dental public health programs, and will also have an understanding of the protocols through which health policies are developed and regulated. To handle oral health programs and presuppose a leadership role in public health, a specialist must acquire methodological skills to understand and manage population health in a variety of settings.

According to World Health Organization, oral health is essential to general health and quality of life. It is a state of being free from mouth and facial pain, oral and throat cancer, oral infection and sores, periodontal (gum) disease, tooth decay, tooth loss, and other diseases and disorders that limit an individual's capacity in biting, chewing, smiling speaking and psychosocial well-being. Public health solutions for oral diseases are most effective when they are integrated with those for other chronic diseases and with national public health programs. The WHO Global Oral Health Program aligns its work with the strategy of chronic disease prevention and health promotion. Emphasis is put on developing global policies in oral health promotion and oral disease prevention.

Knowledge to health policies and management principles will lead to develop next generation oral healthcare workers with analytical attitude for reviewing the problem and focused approach for the management of the disease. The next generation oral healthcare workers will be recognized with term 'Oral Physicians'.

Rajiv Saini

Editor-in-Chief

International Journal of Experimental Dental Science

Associate Professor

Department of Periodontology and Oral Implantology Pravara Institute of Medical Sciences, Loni Ahmednagar, Maharashtra, India 